\title{
COMPLEX OSCILLATION OF SOLUTIONS OF A THIRD ORDER ODE
}

\section{Aimo Hinkkanen, Katsuya Ishizaki, Ilpo Laine and Kin Y. Li}

\author{
University of Illinois at Urbana-Champaign, Department of Mathematics \\ 1409 W. Green Street, Urbana, Illinois 61801-2975, U.S.A.; aimo@math.uiuc.edu \\ The Open University of Japan, Faculty of Liberal Arts \\ Mihama-ku, Chiba, Japan; ishizaki@ouj.ac.jp \\ University of Eastern Finland, Department of Physics and Mathematics \\ P.O. Box 111, FI-80101 Joensuu, Finland; ilpo.laine@uef.fi \\ Hong Kong University of Science and Technology, Department of Mathematics \\ Clear Water Bay, Kowloon, Hong Kong; makyli@ust.hk
}

\begin{abstract}
We solve a determinant problem related to a third order complex linear differential equation studied by Chiang, Laine and Wang. As a consequence, a simple procedure to explicit determination of the corresponding solutions is presented.
\end{abstract}

\section{Introduction}

For complex differential equations, there is an interesting line of investigations on the distribution of the zeros of the meromorphic solutions. If a non-constant meromorphic function $f(z)$ has the zeros $z_{1}, z_{2}, \ldots$ (counted according to multiplicity and ordered by non-decreasing moduli), the exponent of convergence $\lambda(f)$ of $f$ is defined as $\inf \left\{\lambda \geq 0: \sum_{z_{j} \neq 0} 1 /\left|z_{j}\right|^{\lambda}<\infty\right\}$ if there is a real number $\lambda>0$ such that the series $\sum_{z_{j} \neq 0} 1 /\left|z_{j}\right|^{\lambda}$ converges, and $\lambda(f)=\infty$ otherwise. The oscillation theory of complex differential equations (see [3] and [11]) investigates the exponents of convergence of the solutions.

In [4], Bank and Laine studied the oscillation theory of certain second order differential equations with entire periodic coefficients and found explicit representations for the solutions with finite exponents of convergence.

As a concrete special case, Bank, Laine and Langley [5] proved the following result, see also [6]:

Theorem 1.1. Let $K$ be a non-zero complex number. The equation

$$
f^{\prime \prime}+\left(e^{z}-K\right) f=0
$$

admits a non-trivial solution $f$ that satisfies $\lambda(f)<\rho(f)=+\infty$ if, and only if,

$$
K=\frac{(2 n+1)^{2}}{16}
$$

for some non-negative integer $n$, and

$$
f(z)=\psi\left(e^{z / 2}\right) \exp \left(d e^{z / 2}-\frac{2 n+1}{4} z\right)
$$

where $d^{2}+4=0$ and $\psi(\zeta)=\sum_{j=0}^{n} c_{j} \zeta^{j}$.

https://doi.org/10.5186/aasfm.2020.4527

2010 Mathematics Subject Classification: Primary 34M10; Secondary 15A15.

Key words: Complex oscillation theory, exponent of convergence, special determinants.

The second author has been partially supported by the President of the Open University of Japan Grant. 
Actually, it is well known [11], [6] that the case $K=\frac{(2 n+1)^{2}}{16}$ above determines two linearly independent solutions $f_{ \pm}$with $d_{ \pm}= \pm 2 i$ and the polynomial $\psi_{ \pm}\left(e^{z / 2}\right)$ as described in Theorem 1.1. The true nature of the polynomials $\psi_{ \pm}$has been described in terms of special functions by Chiang and Ismail, see [7]:

Theorem 1.2. Given $K=(2 n+1)^{2} / 16$ with $n \geq 0, n \in \mathbf{Z}$, the equation

$$
f^{\prime \prime}+\left(e^{z}-K\right) f=0
$$

admits two linearly independent solutions

$$
f_{ \pm}(z)=\theta_{n}\left(\mp 2 i e^{z / 2}\right) \exp \left( \pm 2 i e^{z / 2}-\frac{2 n+1}{4} z\right)
$$

where $\theta_{n}(\zeta)$ is the reverse Bessel polynomial of degree $n$.

As for the notion of reverse Bessel polynomials, see [9] and [7]. Theorem 1.2 here is a special case of a more general result in [7], Theorem 1.2.

We assume that the reader is familiar with the notation and basic results of the Nevanlinna value distribution theory as given in [10]. In [8], Theorem 1, Chiang, Laine, and Wang established a somewhat similar result concerning the oscillation theory of a third order linear differential equation with entire periodic coefficients using the Nevanlinna theory:

Theorem 1.3. Let $K \in \mathbf{C}$ and suppose that

$$
f^{\prime \prime \prime}-K f^{\prime}+e^{z} f=0
$$

admits a non-trivial solution $f$ such that

$$
\log ^{+} N(r, 1 / f)=o(r) \quad \text { as } r \rightarrow \infty .
$$

Then there exist integers $r$ and $s$ such that $r+s \geq 0$ and

$$
K=\frac{(r+s+1)^{2}}{9} .
$$

Moreover, if $n=r+s>0$, then $n$ satisfies the following tridiagonal $(n+1) \times(n+1)$ determinant condition:

$$
\operatorname{det} \mathbf{A}=0,
$$

where the non-zero diagonals of $\mathbf{A}$ are determined by

$$
\begin{cases}a_{j, j-1}:=(j-1) j(j+1)-2 j n-j n^{2}, & j=1, \ldots, n, \\ a_{j, j}:=-3 j(j+1)+2 n+n^{2}, & j=0, \ldots, n, \\ a_{j, j+1}:=3(j+1), & j=0, \ldots, n-1 .\end{cases}
$$

Furthermore, $f$ admits one of the following representations:

$$
f_{i}(z)=e^{-(s+1) z / 3} \psi\left(e^{z / 3}\right) \exp \left(c_{i} e^{z / 3}\right),
$$

where $c_{i}^{3}+27=0, i=1,2,3$, and

$$
\psi(\zeta)=\sum_{j=-r}^{s} d_{j} \zeta^{j}, \quad d_{-r} d_{s} \neq 0 .
$$

Conversely, suppose $K$ takes the form (1.3) and $n=r+s>0$ and $n$ satisfies (1.4) and (1.5). Then there exists a rational function of the form (1.7) such that the three functions defined by (1.6) are linearly independent solutions of (1.1), each with exponent of convergence $\lambda\left(f_{i}\right) \leq 1$ for $i=1,2,3$. 
Remark. Theorem 1.3 above contains a slight inaccuracy. Namely, in the representations $f_{i}(z)$ in (1.6), the rational component $\psi$ actually depends on $i, i=1,2,3$. Therefore, (1.6) should in fact be written as

$$
f_{i}(z)=e^{-(s+1) z / 3} \psi_{i}\left(e^{z / 3}\right) \exp \left(c_{i} e^{z / 3}\right),
$$

and similarly (1.7) as

$$
\psi_{i}(\zeta)=\sum_{j=-r}^{s} d_{i, j} \zeta^{j}, \quad d_{i,-r} d_{i, s} \neq 0 .
$$

The converse conclusion in Theorem 1.3 then claims the existence of three rational functions of the form (1.9) that determine by (1.8) three linearly independent solutions of (1.1). For a new, more compact formulation of Theorem 1.3, see Theorem 5.1 below.

Remark. As seen in Theorem 5.1 below, the core of $\psi_{i}(\zeta)$ may be expressed as polynomials of $\zeta$. It is possible that these polynomials may be expressed in terms of some special functions, as was the case in Theorem 1.1 above.

In [8], Chiang, Laine and Wang expressed the conjecture that the determinant condition $\operatorname{det} \mathbf{A}=0$ in (1.4) and (1.5), might be equivalent to $n \not \equiv 2(\bmod 3)$, meaning that $K$ would be a ninth of a perfect square, but not an integer. This conjecture has remained open, although it has been verified numerically up to $n=$ 1000, thanks to S. H. Lui at the Hong Kong University of Science and Technology. We also thank Y.-M. Chiang (Hong Kong University of Science and Technology) for his active participation in several discussions about this problem.

The key result in this paper is now to prove this conjecture:

Theorem 1.4. The determinant condition $\operatorname{det} \mathbf{A}=0$ in (1.4) and (1.5), holds if, and only if, $n \neq \equiv 2(\bmod 3)$.

In what follows, if we wish to emphasize the dimension $n+1$, we denote $\mathbf{A}$ by $\mathbf{A}_{n}$. To give a feeling for the determinants $D_{n}:=\operatorname{det} \mathbf{A}_{n}$, note that if we adopt $N=n(n+2)$ as a variable in (1.5), then $D_{n}$ is a non-constant polynomial in $N$ of degree $n+1$. The first determinants $D_{n}$, for $n=0, \ldots, 6$, may now be calculated to obtain

$$
\begin{aligned}
& D_{0}=N, D_{1}=N(N-3), D_{2}=N(N-3)(N-6), \\
& D_{3}=N(N-3)(N-15) N, D_{4}=N(N-3)(N-15)(N-24)(N+12), \\
& D_{5}=N(N-3)(N-15)(N-24)\left(N^{2}-3 N-1080\right), \\
& D_{6}=N(N-3)(N-15)(N-24)(N-48)\left(N^{2}+27 N-1890\right) .
\end{aligned}
$$

\section{Transformation of A}

In what follows, we first apply elementary linear algebra, see, e.g., [12], to transform A by multiplication of finitely many elementary matrices to an upper triangular matrix B. Then it is clear, by the elementary matrix transformations defined next, that $\operatorname{det} \mathbf{A} \neq 0$ if, and only if, all diagonal elements of $\mathbf{B}$ are non-vanishing.

As for the transformations, first denote by $P_{i}(c)$ a matrix obtained from the unit matrix by multiplying the $i$-th diagonal component with a number $c \neq 0$. Multiplication of a matrix $A$ by $P_{i}(c)$ on the left then means to multiply by $c$ the elements of the $i$-th row of $A$. Another elementary matrix $P_{i j}(c)$, to be needed below, is defined by setting $i, j$-component as $c$ for $i \neq j$, and all other components 0 except the diagonal 
components which are equal to 1 . Multiplication of a matrix $A$ by $P_{i, j}(c)$ on the left then means to add $c$ times the $j$-th row of $A$ to the $i$-th row of $A$.

We now consider an $(n+1) \times(n+1)$ tridiagonal matrix $A=\left(a_{i, j}\right)$ such that $a_{i j}=0$, whenever $|i-j| \geq 2$ :

$$
A=\left(\begin{array}{ccccccc}
a_{0,0} & a_{0,1} & 0 & & & & \\
a_{1,0} & a_{1,1} & a_{1,2} & 0 & & 0 & \\
0 & a_{2,1} & a_{2,2} & a_{2,3} & 0 & & \\
& 0 & a_{3,2} & a_{3,3} & \ddots & \ddots & \\
& & 0 & \ddots & \ddots & \ddots & 0 \\
& 0 & & \ddots & \ddots & \ddots & a_{n-1, n} \\
& & & & 0 & a_{n, n-1} & a_{n, n}
\end{array}\right),
$$

with $a_{0,0} \neq 0$. By means of elementary matrix multiplications defined above, we transform $A$ to an upper triangular matrix $A_{n}$ as follows:

As the first step,

$$
\begin{aligned}
A \rightarrow A_{1} & =P_{1,0}\left(-a_{1,0}\right) P_{1}\left(a_{0,0}\right) A \\
& =\left(\begin{array}{ccccccc}
a_{0,0} & a_{0,1} & 0 & & & & \\
0 & a_{1,1} a_{0,0}-a_{0,1} a_{1,0} & a_{1,2} a_{0,0} & 0 & & 0 & \\
0 & a_{2,1} & a_{2,2} & a_{2,3} & 0 & & \\
& 0 & a_{3,2} & a_{3,3} & \ddots & \ddots & \\
& 0 & \ddots & \ddots & \ddots & 0 \\
& 0 & & \ddots & \ddots & \ddots & a_{n-1, n} \\
& & & & 0 & a_{n, n-1} & a_{n, n}
\end{array}\right) .
\end{aligned}
$$

For what follows, we write $a_{0,0}=\beta_{0,0}$ and $a_{1,1} a_{0,0}-a_{0,1} a_{1,0}=\beta_{1,1}$. Then

$$
A_{1}=\left(\begin{array}{ccccccc}
\beta_{0,0} & a_{0,1} & 0 & & & & \\
0 & \beta_{1,1} & a_{1,2} \beta_{0,0} & 0 & & 0 & \\
0 & a_{2,1} & a_{2,2} & a_{2,3} & 0 & & \\
& 0 & a_{3,2} & a_{3,3} & \ddots & \ddots & \\
& & 0 & \ddots & \ddots & \ddots & 0 \\
& 0 & & \ddots & \ddots & \ddots & a_{n-1, n} \\
& & & & 0 & a_{n, n-1} & a_{n, n}
\end{array}\right) .
$$

As for the next step, writing $\beta_{11} a_{22}-a_{21} a_{12} \beta_{00}=\beta_{22}$, we obtain

$$
\begin{aligned}
& A_{1} \rightarrow A_{2}=P_{2,1}\left(-a_{2,1}\right) P_{2}\left(\beta_{1,1}\right) A_{1} \\
& =\left(\begin{array}{ccccccc}
\beta_{0,0} & a_{0,1} & 0 & & & & \\
0 & \beta_{1,1} & a_{1,2} \beta_{0,0} & 0 & & 0 & \\
0 & 0 & \beta_{1,1} a_{2,2}-a_{2,1} a_{1,2} \beta_{0,0} & a_{2,3} \beta_{1,1} & 0 & & \\
& & & & 1 & & \\
& 0 & a_{3,2} & a_{3,3} & \ddots & \ddots & \\
& & 0 & \ddots & \ddots & \ddots & 0 \\
& 0 & & \ddots & \ddots & \ddots & a_{n-1, n} \\
& & & & 0 & a_{n, n-1} & a_{n, n}
\end{array}\right)
\end{aligned}
$$




$$
=\left(\begin{array}{ccccccc}
\beta_{0,0} & a_{0,1} & 0 & & & & \\
0 & \beta_{1,1} & a_{1,2} \beta_{0,0} & 0 & & 0 & \\
0 & 0 & \beta_{2,2} & a_{2,3} \beta_{1,1} & 0 & & \\
& 0 & a_{3,2} & a_{3,3} & \ddots & \ddots & \\
& & 0 & \ddots & \ddots & \ddots & 0 \\
& 0 & & \ddots & \ddots & \ddots & a_{n-1, n} \\
& & & & 0 & a_{n, n-1} & a_{n, n}
\end{array}\right) .
$$

We now continue the process inductively by

$$
\beta_{j+1, j+1}=\beta_{j, j} a_{j+1, j+1}-a_{j+1, j} a_{j, j+1} \beta_{j-1, j-1}, \quad j=1,2, \ldots, n-1
$$

with $\beta_{0,0}=a_{0,0} \neq 0$ and $\beta_{1,1}=\beta_{0,0} a_{1,1}-a_{0,1} a_{1,0}$, to obtain

$$
B=\left(\begin{array}{ccccccc}
\beta_{0,0} & a_{0,1} & 0 & & & & 0 \\
0 & \beta_{1,1} & a_{1,2} \beta_{0,0} & 0 & & & \\
0 & 0 & \beta_{2,2} & a_{2,3} \beta_{1,1} & 0 & & \\
& 0 & 0 & \beta_{3,3} & \ddots & \ddots & \\
& & 0 & \ddots & \ddots & \ddots & 0 \\
& 0 & & \ddots & \ddots & \ddots & a_{n-1, n} \beta_{n-1, n-1}
\end{array}\right) .
$$

We now specify $A$ to be our original tridiagonal matrix $\mathbf{A}_{n}$ defined in (1.5), where $n$ is a fixed integer. Define as $\mathbf{B}$ the transformed matrix, obtained from $A$ for this special case $A=\mathbf{A}_{n}$. By (1.5), we have $\beta_{0,0}=n(n+2)$ and

$$
\begin{aligned}
\beta_{1,1} & =\beta_{0,0} a_{1,1}-a_{0,1} a_{1,0} \\
& =n(n+2)\left(-6+2 n+n^{2}\right)+\left(2 n+n^{2}\right) \cdot 3 \\
& =(n-1) n(n+2)(n+3) .
\end{aligned}
$$

Defining $N:=\beta_{0,0}=n(n+2)$, we have $\beta_{1,1}=N(N-3)$ by (2.4). Making use of $N$, we may write $(1.5)$ as

$$
\begin{cases}a_{j, j-1}:=(j-1) j(j+1)-j N, & j=1, \ldots, n, \\ a_{j, j}:=-3 j(j+1)+N, & j=0, \ldots, n, \\ a_{j, j+1}:=3(j+1), & j=0, \ldots, n-1 .\end{cases}
$$

It follows from $\beta_{0,0}=N, \beta_{1,1}=N(N-3),(2.2)$ and (2.5) that $\beta_{j, j}, j=0,1, \ldots, n$ are polynomials in $N$. Furthermore, we have

Lemma 2.1. Let $\beta_{j, j}, j=0,1,2 \ldots$ be defined by $(2.2)$ with $\beta_{0,0}=N$ and $\beta_{1,1}=N(N-3)$. Then $\beta_{j, j}, j=0,1,2 \ldots$ are polynomials in $N$ of degree $j+1$, and coefficient of $N^{j+1}$ in $\beta_{j, j}$ equals 1 .

Proof. Assume that the assertion holds for the cases $j$ and $j-1$. By (2.5), $\beta_{j, j} a_{j+1, j+1}$ is a polynomial in $N$ of degree $j+2$ and the coefficient of $N^{j+2}$ is 1 . Moreover, $-a_{j+1, j} a_{j, j+1} \beta_{j-1, j-1}$ is a polynomial of degree $j+1$ in $N$. This implies that $\beta_{j+1, j+1}$ is a polynomial of degree $j+2$ in $N$, and the coefficient of $N^{j+2}$ equals 1 by $(2.2)$, proving the lemma by induction. 


\section{Proof of Theorem 1.4, the first part}

We first prove the following proposition, which is the easy part in proving Theorem 1.4:

Proposition 3.1. If $\operatorname{det} \mathbf{A}=\operatorname{det} \mathbf{B}=0$, then $n \not \equiv 2 \quad(\bmod 3)$.

Proof. We will show that $n \equiv 2$, (or -1$)(\bmod 3) \operatorname{implies} \operatorname{det} \mathbf{A} \neq 0$. Suppose that $n \equiv 2(\bmod 3)$. Then there exists an integer $k$ such that $n=3 k-1$, which gives

$$
\beta_{0,0} \equiv m=n(n+2)=(3 k-1)(3 k+1)=9 k^{2}-1 \equiv-1 \quad(\bmod 3)
$$

and

$$
\begin{aligned}
\beta_{1,1} & =\beta_{00} a_{11}-a_{01} a_{10}=(3 k-2)(3 k-1)(3 k+1)(3 k+2) \\
& =\left(9 k^{2}-4\right)\left(9 k^{2}-1\right)=81 k^{4}-45 k^{2}+4 \equiv 1 \quad(\bmod 3) .
\end{aligned}
$$

We complete the proof by induction. Assume that for $j \leq \ell, \beta_{j, j} \equiv-1(\bmod 3)$ if $j$ is even, and $\beta_{j, j} \equiv 1(\bmod 3)$ if $j$ is odd. We note that $a_{j, j}=-3 j(j+1)+m=-1$ $(\bmod 3)$. In case $\ell$ is odd, we have some integers $k_{1}, \ldots, k_{4}$ such that

$$
\begin{aligned}
\beta_{\ell+1, \ell+1} & =\beta_{\ell, \ell} a_{\ell+1, \ell+1}-a_{\ell+1, \ell} a_{\ell, \ell+1} \beta_{\ell-1, \ell-1} \\
& =\left(3 k_{1}+1\right)\left(3 k_{2}-1\right)-3 k_{3}=3 k_{4}-1 \equiv-1 \quad(\bmod 3) .
\end{aligned}
$$

Similarly, we obtain $\beta_{\ell+1, \ell+1} \equiv 1(\bmod 3)$ when $\ell$ is even. This gives that $\beta_{j, j}$, $j=0,1, \ldots, n$ do not vanish in (2.3), and hence $\operatorname{det} \mathbf{A} \neq 0$.

\section{Proof of Theorem 1.4, the converse part}

4.1. Notations and definitions. We define the set $E$ of certain integers by

$$
E=\{n \in \mathbf{Z}: n \geq 0, n \not \equiv 2(\bmod 3)\} .
$$

Thus $E=\{0,1,3,4,6,7, \ldots\}$.

We set $\alpha_{-1}=\alpha_{-1}(N)=1$ and define for $j \geq 0$,

$$
\alpha_{j}=\alpha_{j}(N)=\prod_{0 \leq k \leq j, k \in E}(N-k(k+2)) .
$$

Thus $\alpha_{j}$ is a polynomial of degree $[2(j+2) / 3]$ in $N$ (where for a real number $x$, the notation $[x]$ means the largest integer $\leq x)$ and

$$
\begin{aligned}
& \alpha_{0}=N, \alpha_{1}=\alpha_{2}=N(N-3), \alpha_{3}=N(N-3)(N-15), \\
& \alpha_{4}=\alpha_{5}=N(N-3)(N-15)(N-24), \\
& \alpha_{6}=N(N-3)(N-15)(N-24)(N-48) .
\end{aligned}
$$

We write $\operatorname{det} \mathbf{A}_{n}$ in the form

$$
\operatorname{det} \mathbf{A}_{n}=\alpha_{n} Q_{n}(N) \text {. }
$$

Then $Q_{n}(N)$ is a rational function of $N$. We will prove later that $Q_{n}$ is a polynomial in $N$, but as long as we do not know that, we should think of $Q_{n}$ as a rational function. Since $\alpha_{n}$ is a polynomial in $N$ (and so is $\operatorname{det} \mathbf{A}_{n}$ ), there are at most finitely many real values of $N$ for which $Q_{n}(N)$ is not a well-defined real number.

From the formulas for $D_{n}$ above we see that

$$
\begin{aligned}
& Q_{0}=Q_{1}=1, Q_{2}=N-6, Q_{3}=N, Q_{4}=N+12, \\
& Q_{5}=N^{2}-3 N-1080, Q_{6}=N^{2}+27 N-1890 .
\end{aligned}
$$


We next derive recursion formulas for the functions $D_{j}$ and after that for the functions $Q_{j}$.

The last row of $\mathbf{A}_{n}$ has only two non-zero elements, and they are the last two elements. Developing $\operatorname{det} \mathbf{A}_{n}$ according to the last row, we get

$$
D_{n}=\operatorname{det} \mathbf{A}_{n}=a_{n n} D_{n-1}-a_{n, n-1} \operatorname{det} B,
$$

where the matrix $B$ is obtained by deleting from $\mathbf{A}$ the last row and the second last column. The last row of $B$ has only two non-zero elements, and they are the last two elements, namely, $a_{n-1, n-2}$ and $a_{n-1, n}$. Developing det $B$ according to the last row of $B$, we get

$$
\operatorname{det} B=a_{n-1, n} D_{n-2}-a_{n-1, n-2} \operatorname{det} B^{\prime},
$$

where the matrix $B^{\prime}$ is obtained by deleting from $B$ the last row and the second last column. Thus the last column of $B^{\prime}$ is a zero column, so that $\operatorname{det} B^{\prime}=0$. This yields

$$
D_{n}=a_{n n} D_{n-1}-a_{n, n-1} a_{n-1, n} D_{n-2} .
$$

Substituting $D_{j}=\alpha_{j} Q_{j}(N)$ for $j \in\{n-2, n-1, n\}$ into this equation, and noting that $a_{n n}=N-3 n(n+1), a_{n, n-1}=(n-1) n(n+1)-n N$, and $a_{n-1, n}=3 n$, we obtain

$$
\begin{aligned}
\alpha_{n} Q_{n}(N)= & (N-3 n(n+1)) \alpha_{n-1} Q_{n-1}(N) \\
& -n((n-1)(n+1)-N) 3 n \alpha_{n-2} Q_{n-2}(N) .
\end{aligned}
$$

We divide the further development of this formula into three cases depending on the residue class of $n$ modulo 3 .

Suppose that $n=3 k$ for some integer $k$. Then

$$
\alpha_{n-1} / \alpha_{n}=\alpha_{n-2} / \alpha_{n}=1 /(N-n(n+2)) .
$$

Thus by (4.5),

$$
(N-n(n+2)) Q_{n}=(N-3 n(n+1)) Q_{n-1}+3 n^{2}\left(N-n^{2}+1\right) Q_{n-2} .
$$

This is compatible with all the functions $Q_{j}$ being rational functions of $N$. We note that if $Q_{n-2}$ and $Q_{n-1}$ are polynomials, then so is $Q_{n}$ if, and only if, the right hand side of (4.6) is divisible by $N-n(n+2)$, which is true if, and only if, the right hand side of (4.6) is equal to zero when $N=n(n+2)$. This is indeed the case, as we will show later, but right now we do not know that so that we only think of $Q_{n}$ as a rational function of $N$.

Suppose that $n=3 k+1$ for some integer $k$. Then

$$
\frac{\alpha_{n-1}}{\alpha_{n}}=\frac{1}{N-n(n+2)}, \quad \frac{\alpha_{n-2}}{\alpha_{n}}=\frac{1}{(N-n(n+2))(N-(n-1)(n+1))} .
$$

Thus by (4.5),

$$
(N-n(n+2)) Q_{n}=(N-3 n(n+1)) Q_{n-1}+3 n^{2} Q_{n-2} .
$$

The comments regarding the possibility of the $Q_{j}$ being polynomials that were made in the case $n=3 k$ apply also when $n=3 k+1$.

Suppose that $n=3 k+2$ for some integer $k$. Then

$$
\alpha_{n-1} / \alpha_{n}=1, \alpha_{n-2} / \alpha_{n}=\frac{1}{N-(n-1)(n+1)} .
$$

Thus by (4.5),

$$
Q_{n}=(N-3 n(n+1)) Q_{n-1}+3 n^{2} Q_{n-2}
$$


So when $n=3 k+2$, if $Q_{n-2}$ and $Q_{n-1}$ are polynomials, then so is $Q_{n}$, and there are no extra conditions.

We have now established the recursion formulas for the functions $D_{j}$ and the functions $Q_{j}$. We collect the results of this subsection together in the following lemma.

Lemma 4.1. Suppose that $n \geq 1$ and write $D_{n}=\operatorname{det} \boldsymbol{A}_{n}$, so that $D_{n}$ is a polynomial in the variable $N$. With $\alpha_{n}$ given by (4.2) (where $E$ is as in (4.1)), write $D_{n}=\alpha_{n} Q_{n}$ where $Q_{n}$ is a rational function of $N$. Then $Q_{0}(N)=Q_{1}(N)=1$, and the functions $Q_{n}$ satisfy the following recursion formulas. If $n=3 k$ for some integer $k$, then

$$
(N-n(n+2)) Q_{n}=(N-3 n(n+1)) Q_{n-1}+3 n^{2}\left(N-n^{2}+1\right) Q_{n-2} .
$$

If $n=3 k+1$ for some integer $k$, then

$$
(N-n(n+2)) Q_{n}=(N-3 n(n+1)) Q_{n-1}+3 n^{2} Q_{n-2} .
$$

If $n=3 k+2$ for some integer $k$, then

$$
Q_{n}=(N-3 n(n+1)) Q_{n-1}+3 n^{2} Q_{n-2}
$$

4.2. Formulas for the solution $\mathbf{v}$ of $\mathbf{A} \mathbf{v}=\mathbf{0}$. Suppose that $\mathbf{v}=\left(x_{0}, x_{1}, \ldots\right.$, $\left.x_{n}\right)$ and $\mathbf{A v}=0$. We may express the equations to be satisfied by $x_{0}, x_{1}, \ldots, x_{n}$ as follows. Considering the $j^{\text {th }}$ component of the equation $\mathbf{A v}=0$, we obtain

$$
a_{j, j-1} x_{j-1}+a_{j j} x_{j}+a_{j, j+1} x_{j+1}=0 .
$$

When $j=0$, this reads

$$
N x_{0}+3 x_{1}=0
$$

Thus

$$
x_{1}=-\frac{N}{3} x_{0}
$$

When $j=n$, this reads

$$
((n-1) n(n+1)-n N) x_{n-1}+(N-3 n(n+1)) x_{n}=0 .
$$

In the special case when $N=n(n+2)$, this reads

$$
x_{n}=-x_{n-1} .
$$

When $1 \leq j \leq n-1$, we get the equation

$$
((j-1) j(j+1)-j N) x_{j-1}+(N-3 j(j+1)) x_{j}+3(j+1) x_{j+1}=0 .
$$

Using the equation (4.17) inductively, starting with $j=1$, we can proceed up to $j=n-1$ to get each $x_{j}$ as $x_{0}$ multiplied by a constant depending on $j$. This is also true for $x_{1}$ by (4.14). It is then clear that the equation $\mathbf{A v}=\mathbf{0}$ (when $N=n(n+2)$ ) has a non-zero solution $\mathbf{v}$ if, and only if, the values of $x_{n-1}$ and $x_{n}$ so obtained satisfy (4.16) identically, that is, for all values of $x_{0}$.

When we solve for $x_{j+1}$ from (4.17), we are dividing by the number $3(j+1)$ that is independent of $N$. Hence it is clear that regarding $N$ as a variable, we obtain each $x_{j}$ for $1 \leq j \leq n$ as a polynomial in $N$ (where we have not used (4.15) yet).

We further note that when we use $N$ as a variable, instead of taking for $N$ a specific value that depends on $n$, the formulas that we obtain for $x_{j}$ are independent of $n$. That is, if we specify any value for $j$, and if we then choose any value for $n$ with $n \geq j$, we get the same formula for $x_{j}$ regardless of the choice of $n$. 
We set $\beta_{-1}=1$, and for $j \geq 0$, we define

$$
\beta_{j}=\prod_{k=0}^{j}(3(k+1))=3^{j+1}((j+1) !) .
$$

Recall that $\alpha_{j}$ is defined by (4.2) and that $\alpha_{-1}=1$.

We now write $x_{j}$ for $1 \leq j \leq n$ in the form

$$
x_{j}=(-1)^{j} \frac{\alpha_{j-1}}{\beta_{j-1}} x_{0} P_{j-1}(N) .
$$

As is compatible with the rest of this notation, we set $P_{-1}(N)=1$. Then (4.19) is valid for $j=0$ also. It then follows that $P_{j}(N)$, for $-1 \leq j \leq n-1$, is a rational function of $N$. We will prove later that $P_{j}=Q_{j}$.

From (4.14) we obtain

$$
P_{0}(N)=1 \text {. }
$$

We next derive recursion formulas for the functions $P_{j}$. Substituting (4.19) into (4.17), we obtain

$$
\begin{aligned}
& -3(j+1)(-1)^{j+1} \frac{\alpha_{j}}{\beta_{j}} x_{0} P_{j}(N) \\
& =((j-1) j(j+1)-j N)(-1)^{j-1} \frac{\alpha_{j-2}}{\beta_{j-2}} x_{0} P_{j-2}(N) \\
& \quad+(N-3 j(j+1))(-1)^{j} \frac{\alpha_{j-1}}{\beta_{j-1}} x_{0} P_{j-1}(N) .
\end{aligned}
$$

For this to be valid when $x_{0} \neq 0$, we must have

$$
\frac{\alpha_{j}}{\beta_{j}} P_{j}=\frac{N-3 j(j+1)}{3(j+1)} \frac{\alpha_{j-1}}{\beta_{j-1}} P_{j-1}+\frac{N-j^{2}+1}{3(j+1)} j \frac{\alpha_{j-2}}{\beta_{j-2}} P_{j-2} .
$$

We again divide the further development of this formula into three cases depending on the residue class of $j$ modulo 3 .

Suppose that $j=3 k$ for some integer $k$. As we saw before, then

$$
\alpha_{j-1} / \alpha_{j}=\alpha_{j-2} / \alpha_{j}=1 /(N-j(j+2)) \text {. }
$$

Also

$$
\frac{\beta_{j-1}}{\beta_{j}}=\frac{1}{3(j+1)}, \frac{\beta_{j-2}}{\beta_{j}}=\frac{1}{9 j(j+1)} .
$$

Thus by (4.22),

$$
(N-j(j+2)) P_{j}=(N-3 j(j+1)) P_{j-1}+3 j^{2}\left(N-j^{2}+1\right) P_{j-2} .
$$

Suppose that $j=3 k+1$ for some integer $k$. As we saw before, then

$$
\frac{\alpha_{j-1}}{\alpha_{j}}=\frac{1}{N-j(j+2)}, \frac{\alpha_{j-2}}{\alpha_{j}}=\frac{1}{(N-j(j+2))(N-(j-1)(j+1))} .
$$

Also

$$
\frac{\beta_{j-1}}{\beta_{j}}=\frac{1}{3(j+1)}, \frac{\beta_{j-2}}{\beta_{j}}=\frac{1}{9 j(j+1)} .
$$

Thus by (4.22),

$$
(N-j(j+2)) P_{j}=(N-3 j(j+1)) P_{j-1}+3 j^{2} P_{j-2} .
$$


When $j=3 k$ or $j=3 k+1$, the comments regarding the possibility of the various $P_{\ell}$ being polynomials are similar to the comments that were made for the $Q_{\ell}$.

Suppose that $j=3 k+2$ for some integer $k$. As we saw before, then

$$
\alpha_{j-1} / \alpha_{j}=1, \alpha_{j-2} / \alpha_{j}=\frac{1}{N-(j-1)(j+1)} .
$$

Also

$$
\frac{\beta_{j-1}}{\beta_{j}}=\frac{1}{3(j+1)}, \frac{\beta_{j-2}}{\beta_{j}}=\frac{1}{9 j(j+1)} .
$$

Thus by (4.22),

$$
P_{j}=(N-3 j(j+1)) P_{j-1}+3 j^{2} P_{j-2} .
$$

When $j=3 k+2$, the function $P_{j}$ is a polynomial if both $P_{j-1}$ and $P_{j-2}$ are polynomials, without any extra conditions.

We have now established the recursion formulas for the functions $P_{j}$. We have found $P_{0}$ before (we have $P_{0}=1$ ). With $P_{-1}=1,(4.19)$ is valid with $j=0$. We calculate a few more functions $P_{j}$. Taking $j=1$ in (4.28), we obtain

$$
(N-3) P_{1}=(N-6) P_{0}+3 P_{-1}=N-6+3=N-3,
$$

so that

$$
P_{1}=1
$$

Taking $j=2$ in (4.31), we obtain

$$
P_{2}=N-6 .
$$

Continuing in the same way, we get

$$
P_{3}=N, P_{4}=N+12 .
$$

We see that

$$
P_{j}(N)=Q_{j}(N)
$$

at least for $0 \leq j \leq 4$. We will see later that (4.32) is valid for all $j \geq 1$.

By now we have seen that the equation $\mathbf{A} \mathbf{v}=0$ implies that for $1 \leq j \leq n$, each $x_{j}$ is a certain multiple of $x_{0}$. In addition the equation $\mathbf{A} \mathbf{v}=0$ for $\mathbf{v} \neq 0$ implies that (4.15) must be valid also when $x_{0} \neq 0$, and for $N=n(n+2),(4.15)$ is equivalent to (4.16). Since $x_{n-1}$ and $x_{n}$ have already been determined, this imposes a further condition on $P_{n-1}$ and $P_{n-2}$. We now determine that condition when $N=n(n+2)$.

Using (4.19) with $j=n-1$ and $j=n$ and substituting the results into (4.16), we obtain, when $x_{0} \neq 0$,

$$
\frac{\alpha_{n-2}}{\beta_{n-2}} P_{n-2}(N)=\frac{\alpha_{n-1}}{\beta_{n-1}} P_{n-1}(N) .
$$

We require this to be true only when $n$ is of the form $3 k$ or $3 k+1$.

Suppose that $n=3 k$ for some integer $k$, so that $n-1$ is of the form $3 k+2$. Using (4.29) and (4.30) with $j$ replaced by $n-1$ in those formulas, we can write (4.33) in the form

$$
3 n P_{n-2}(N)=P_{n-1}(N) .
$$

Since we want this to be true when $N=n(n+2)$, we require, more precisely, that

$$
3 n P_{n-2}(n(n+2))=P_{n-1}(n(n+2)) \text {. }
$$


Suppose that $n=3 k+1$ for some integer $k$, so that $n-1$ is of the form $3 k$. Using (4.23) and (4.24) with $j$ replaced by $n-1$ in those formulas, we can write (4.33) in the form

$$
3 n P_{n-2}(n(n+2))=(N-(n-1)(n+1)) P_{n-1}(n(n+2)) .
$$

When $N=n(n+2)$, we have

$$
N-(n-1)(n+1)=n(n+2)-(n-1)(n+1)=2 n+1 .
$$

Since we want (4.35) to be true when $N=n(n+2)$, we require, more precisely, that

$$
3 n P_{n-2}(n(n+2))=(2 n+1) P_{n-1}(n(n+2)) \text {. }
$$

We collect the results of this subsection together in the following lemma.

Lemma 4.2. Suppose that $n \geq 1$. Then the equation $\mathbf{A}_{n} \mathbf{v}=0$ has a non-zero solution $\mathbf{v}=\left(x_{0}, x_{1}, \ldots, x_{n}\right)$ if, and only if, with the notation (4.19), the rational functions $P_{j}(N)$ of $N$ satisfy the recursion formulas given below for $1 \leq j \leq n-1$ and, in addition, (4.15) holds (hence (4.16) when $N=n(n+2)$ ). The recursion formulas are as follows. We have $P_{-1}(N)=P_{0}(N)=P_{1}(N)=1$. If $j=3 k$ for some integer $k$, then

$$
(N-j(j+2)) P_{j}=(N-3 j(j+1)) P_{j-1}+3 j^{2}\left(N-j^{2}+1\right) P_{j-2} .
$$

If $j=3 k+1$ for some integer $k$, then

$$
(N-j(j+2)) P_{j}=(N-3 j(j+1)) P_{j-1}+3 j^{2} P_{j-2} .
$$

If $j=3 k+2$ for some integer $k$, then

$$
P_{j}=(N-3 j(j+1)) P_{j-1}+3 j^{2} P_{j-2} \text {. }
$$

For the rest of this lemma, we assume that $N=n(n+2)$. If $n=3 k$ for some integer $k$, then (4.16) is equivalent to

$$
3 n P_{n-2}(n(n+2))=P_{n-1}(n(n+2)) \text {. }
$$

If $n=3 k+1$ for some integer $k$, then (4.16) is equivalent to

$$
3 n P_{n-2}(n(n+2))=(2 n+1) P_{n-1}(n(n+2)) \text {. }
$$

4.3. Connection between the formulas for $\operatorname{det} \mathbf{A}_{\boldsymbol{n}}$ and $\mathrm{v}$. We note that the functions $Q_{j}$ and $P_{j}$ are defined in the same way, no matter which value of $n$ with $n \geq j+1$ is considered to define $Q_{j}$ and $P_{j}$. The following lemma expresses the relationship between $Q_{j}$ and $P_{j}$.

Lemma 4.3. For all $j \geq 0$, we have

$$
P_{j}(N)=Q_{j}(N)
$$

To prove Lemma 4.3, we merely have to note that (4.42) is valid for $j=0$ and $j=1$, and that the recursion formulas for $Q_{j}$ are identical to those for $P_{j}$.

4.4. The final statements to be proved by induction. For $n \geq 1$ with $n \in E$, we define the statement $S(n)$ as follows.

If $n=3 k$ for some integer $k$, then $S(n)$ states that (4.40) holds.

If $n=3 k+1$ for some integer $k$, then $S(n)$ states that (4.41) holds.

We will prove the following result by induction on $n$. Recall that the set $E$ is defined by (4.1).

Lemma 4.4. For every integer $n \geq 1$, all of the following statements are valid: 
(i) For all $j$ with $1 \leq j \leq n$ such that $j \in E$, we have

$$
\operatorname{det} \mathbf{A}_{j}=0
$$

when $N=j(j+2)$.

(ii) For all $j$ with $1 \leq j \leq n-1$, the function $P_{j}(N)$ (and hence also the function $\left.Q_{j}(N)\right)$ is a polynomial in $N$.

(iii) For all $j$ with $1 \leq j \leq n$ such that $j \in E$, the statement $S(j)$ is valid.

Clearly, after Lemma 4.4 has been proved, Theorem 1.4 follows immediately.

4.5. Proof of Lemma 4.4. To be able to use the recursion formulas for $P_{j}$, we need to prove two base cases first. If $n=1$, then (ii) and (iii) of Lemma 4.4 are vacuous, while (i) states that $\operatorname{det} \mathbf{A}_{1}=0$, that is, $D_{1}=0$ when $N=n(n+2)=3$. Since $D_{1}=N(N-3)$, this is true.

As a side-remark, we note that if $n=0$ and $N=n(n+2)=0$, then $D_{0}=N=0$ also. We further know that $P_{0}(N)=Q_{0}(N)=1$ is a polynomial in $N$.

Suppose that $n=2$, so $n \notin E$. Then (i) imposes no further requirements beyond what was demanded for $n=1$. For (ii), we need to know that $P_{1}(N)$ is a polynomial in $N$, which is true since $P_{1}(N) \equiv 1$. The condition (iii) requires that $S(1)$ is true, that is, that (4.41) holds when we take $n=1$ in (4.41). This reads $3 P_{-1}(3)=3 P_{0}(3)$, which is true since $P_{-1}(N) \equiv 1$ and $P_{0}(N) \equiv 1$.

For more convenient notation, suppose that $n \geq 3$ and that the claims of Lemma 4.4 are valid for $n-1$ instead of $n$. For the induction step, we need to prove that these claims are valid for $n$. To do this, we need to prove the following. For (i), if $n \in E$, we need to prove that $\operatorname{det} \mathbf{A}_{n}=0$ when $N=n(n+2)$ (while if $n \notin E$, we do not need to prove anything for (i)). For (ii), we need to prove that $P_{n-1}(N)$ is a polynomial in $N$. For (iii), we need to prove that if $n \in E$, then $S(n)$ is true (while if $n \notin E$, we do not need to prove anything for (iii)).

Let us begin with the proof of (ii), which we divide into three cases according to the residue class of $n$ modulo 3. Thus we are assuming that $P_{\ell}(N)$ is a polynomial in $N$ for $1 \leq \ell \leq n-2$.

Suppose first that $n$ is of the form $n=3 k$, so $n-1=3(k-1)+2$. Write $j=n-1$. Then the formula (4.31) is applicable, and it gives $P_{j}(N)$, that is, $P_{n-1}(N)$, as

$$
P_{j}(N)=(N-3 j(j+1)) P_{j-1}(N)+3 j^{2} P_{j-2}(N) .
$$

By the induction assumption, $P_{j-1}$ and $P_{j-2}$ are polynomials in $N$, so that $P_{j}(N)$ is also trivially a polynomial in $N$.

Suppose next that $n$ is of the form $n=3 k+1$, so $n-1=3 k$. Write $j=n-1$. Then the formula (4.25) is applicable, and it gives $P_{j}(N)$, that is, $P_{n-1}(N)$, as

$$
(N-j(j+2)) P_{j}(N)=(N-3 j(j+1)) P_{j-1}(N)+3 j^{2}\left(N-j^{2}+1\right) P_{j-2}(N) .
$$

To prove that $P_{j}$ is a polynomial is equivalent to proving that the right hand side of (4.45) is equal to zero when $N=j(j+2)$. We write the right hand side of (4.45) as

$$
\begin{aligned}
& (N-j(j+2))\left(P_{j-1}+3 j^{2} P_{j-2}\right) \\
& +(j(j+2)-3 j(j+1)) P_{j-1}+3 j^{2}\left(j(j+2)-j^{2}+1\right) P_{j-2} \\
& =(N-j(j+2))\left(P_{j-1}+3 j^{2} P_{j-2}\right)-j(2 j+1)\left(P_{j-1}-3 j P_{j-2}\right) .
\end{aligned}
$$

Thus we need to prove that for $j=n-1=3 k \in E$, we have

$$
P_{j-1}(j(j+2))=3 j P_{j-2}(j(j+2)) .
$$


Our induction assumption regarding (i) implies that det $\mathbf{A}_{n-1}=0$. Thus the equation $\mathbf{A}_{n-1} \mathbf{v}=0$ has a non-zero solution $\mathbf{v}$, and now (4.40) of Lemma 4.2 implies (4.46) on replacing $n$ by $j$ in (4.40).

Suppose then that $n$ is of the form $n=3 k+2$, so $n-1=3 k+1$. Write $j=n-1$. Then the formula (4.28) is applicable, and it gives $P_{j}(N)$, that is, $P_{n-1}(N)$, as

$$
(N-j(j+2)) P_{j}(N)=(N-3 j(j+1)) P_{j-1}(N)+3 j^{2} P_{j-2}(N) .
$$

To prove that $P_{j}$ is a polynomial is equivalent to proving that the right hand side of (4.47) is equal to zero when $N=j(j+2)$. We write the right hand side of (4.47) as

$$
\begin{aligned}
& (N-j(j+2)) P_{j-1}+(j(j+2)-3 j(j+1)) P_{j-1}+3 j^{2} P_{j-2} \\
& =(N-j(j+2)) P_{j-1}-j\left((2 j+1) P_{j-1}-3 j P_{j-2}\right) .
\end{aligned}
$$

Thus we need to prove that for $j=n-1=3 k+1 \in E$, we have

$$
(2 j+1) P_{j-1}(j(j+2))=3 j P_{j-2}(j(j+2)) .
$$

Our induction assumption regarding (i) implies that det $\mathbf{A}_{n-1}=0$. Thus the equation $\mathbf{A}_{n-1} \mathbf{v}=0$ has a non-zero solution $\mathbf{v}$, and now (4.41) of Lemma 4.2 implies (4.48) on replacing $n$ by $j$ in (4.41). This completes the proof that $P_{n-1}(N)$ is a polynomial in $N$, so that (ii) is proved for $n$.

Next, we prove (i), that is, we prove that $\operatorname{det} \mathbf{A}_{n}=0$ if $n \in E$ and $N=n(n+2)$. For this, we only need to use (4.3) and note that since $P_{n}=Q_{n}$ by Lemma 4.3, also $Q_{n}(N)$ is a polynomial in $N$. Now, since $n \in E$, the product $\alpha_{n}$ contains the factor $N-n(n+2)$, so that $\alpha_{n}=0$ and hence $\operatorname{det} \mathbf{A}_{n}=0$ when $N=n(n+2)$. This proves (i) for $n$.

For (iii), suppose that $n \in E$. By what we have proved already, we know that $\operatorname{det} \mathbf{A}_{n}=0$ when $N=n(n+2)$, so that for this $N$, the equation $\mathbf{A}_{n} \mathbf{v}=0$ has a non-zero solution $\mathbf{v}$. By Lemma 4.2, the equation (4.40) holds if $n=3 k$, and the equation (4.41) holds if $n=3 k+1$. This gives $S(n)$. This completes the proof of Lemma 4.4 and hence also the converse part of Theorem 1.4.

\section{Discussion}

In [2], Bank constructed a method to test the second order linear differential equation

$$
f^{\prime \prime}+A(z) f=0
$$

where $A(z)$ is a non-constant periodic entire function, for the existence of solutions $f$ satisfying $\lambda(f)<\infty$, as well as to construct these solutions explicitly. The method in [2] involves about finding polynomial solutions of four second order linear differential equations. This method has been extended by Baesch [1] to test higher order linear differential equations of type

$$
f^{(n)}+\sum_{j=1}^{n-2} A_{j}(z) f^{(j)}+A_{0}(z) f=0,
$$

where $A_{1}, \ldots, A_{n-2}$ are constants, $n \geq 3$, and $A_{0}$ is a non-constant periodic entire function. In this higher order case, $n^{2}$ linear differential equations of order $n$ have to be tested for polynomial solutions. For a more general consideration where the coefficients are permitted to be rational functions of $e^{z}$, see [13].

Unfortunately, the procedure described in [2], [1] is somewhat difficult to be applied in practice, although it is certainly possible to implement a suitable software package for such a purpose. In the special case of (1.1) with the determinant condition 
(1.4), it is much easier to get an explicit construction of solutions $f$ with $\lambda(f) \leq 1$ from a reformulation of Theorem 1.3:

Theorem 5.1. The linear differential equation (1.1) with $K \in \mathbf{C}$ admits a nontrivial solution $f$ such that

$$
\log ^{+} N(r, 1 / f)=o(r) \text { as } r \rightarrow \infty,
$$

if, and only if,

$$
K=\frac{(n+1)^{2}}{9}
$$

for some integer $n \geq 0$ such that $n \not \equiv 2(\bmod 3)$. Moreover, in this case, three linearly independent such solutions $f$ exist, corresponding to the three complex numbers $c$ such that $c^{3}+27=0$. With $\zeta:=e^{z / 3}$, these solutions $f$ have a representation of the form

$$
f(z)=e^{c \zeta} \zeta^{-1} Q\left(c^{-1} \zeta^{-1}\right)
$$

where $Q$ is a polynomial of degree $n$, independent of $c$. The function $Q(z)$ satisfies the differential equation

$$
z^{4} Q^{\prime \prime \prime}+z^{2}(6 z-3) Q^{\prime \prime}-\left(6 z-3+(n(n+2)-6) z^{2}\right) Q^{\prime}-n(n+2)(z-1) Q=0
$$

Furthermore, with $P_{j}(N)$ defined as in Section 4 , with $\beta_{j}=3^{j+1}((j+1) !)$ defined as in (4.18), and with $\alpha_{j}(N)$ defined as in (4.2), we may write

$$
Q(z)=1+\sum_{j=1}^{n} d_{j} z^{j}
$$

where

$$
d_{j}=(-1)^{j} \frac{\alpha_{j-1}(n(n+2))}{\beta_{j-1}} P_{j-1}(n(n+2)),
$$

for $1 \leq j \leq n$. In particular, $\lambda(f)=0$ for $n=0$ and $\lambda(f)=1$ for $n>0$, in each case for all three choices of $c$.

Remark. Thus the numbers $d_{j}$ are the same as the numbers $x_{j}$ given by (4.19), when taking $x_{0}=1$ and $N=n(n+2)$ in (4.19). By Lemma 4.1 and Lemma 4.3, we may also write

$$
d_{j}=(-1)^{j} \frac{\operatorname{det} \mathbf{A}_{j-1}}{3^{j}(j !)}
$$

where we take $N=n(n+2)$ when computing $\operatorname{det} \mathbf{A}_{j-1}$. Thus it has been useful for us to solve the equation $\mathbf{A v}=0$ not only to prove Theorem 1.4 but also to obtain the coefficients of $Q$ for a more explicit solution of (1.1). In spite of their appearance, the numbers $d_{j}$ are integers, but we will not prove that here.

Proof. Suppose that $n \geq 0, n \in E$, and $K=(n+1)^{2} / 9$. We look for a solution $f$ of (1.1) in the form $f(z)=F\left(c e^{z / 3}\right)$, where $c^{3}=-27$. Substituting this $f$ into (1.1), denoting in the resulting equation the quantity $c e^{z / 3}$ by $t$ and using the fact that $c^{3}=-27$, we obtain for the function $F(t)$ the equation

$$
t^{3} F^{\prime \prime \prime}(t)+3 t^{2} F^{\prime \prime}(t)-n(n+2) t F^{\prime}(t)-t^{3} F(t)=0 .
$$

This equation is independent of $c$, so that for each choice of $c$, a solution $F$ of (5.6) yields a solution $f$ of (1.1). We look for $F$ in the form $F(t)=e^{t} g(1 / t)$. Substituting 
this $F$ into (5.6) and denoting in the resulting equation $1 / t$ by $z$, we obtain for $g(z)$ the equation

$$
z^{3} g^{\prime \prime \prime}+3 z(z-1) g^{\prime \prime}-\frac{3}{z}\left(\frac{g}{z}-g^{\prime}\right)+n(n+2)\left(\frac{g}{z}-z g^{\prime}\right)=0 .
$$

Writing $g(z)=z Q(z)$ we get for the function $Q(z)$ the equation (5.2). Thus with $\zeta=e^{z / 3}$, a solution $Q$ of (5.2) gives the solution $f(z)=e^{c \zeta}(c \zeta)^{-1} Q(1 /(c \zeta))$ of (1.1). Then also $c f$ is a solution of (1.1), and this gives the right hand side of (5.1).

We next verify that (5.2) has a solution $Q$ that is a polynomial. When $n=0$, it is obvious that $Q(z) \equiv 1$ is a solution of (5.2). Suppose that $n \geq 1$ and $n \in E$. Write $N=n(n+2)$. Substituting

$$
Q(z)=\sum_{j=0}^{n} d_{j} z^{j}
$$

into (5.2) we get the recursion formulas for the $d_{j}$. We need to consider the coefficient of $z^{j}$ on the left hand side of (5.2), for $0 \leq j \leq n+1$.

Considering the constant coefficient on the left hand side of (5.2), we get

$$
d_{1}=-(N / 3) d_{0}
$$

The coefficient of $z^{j}$ for $1 \leq j \leq n-1$ yields

$$
3(j+1) d_{j+1}+(N-3 j(j+1)) d_{j}+\left(j^{2}-1-N\right) j d_{j-1}=0 .
$$

The coefficient of $z^{n}$ gives

$$
(N-3 n(n+1)) d_{n}+\left(n^{2}-1-N\right) n d_{n-1}=0,
$$

and since $N=n(n+2)$, this is equivalent to

$$
d_{n}=-d_{n-1} .
$$

The coefficient of $z^{n+1}$ vanishes identically, regardless of the value of $d_{n}$.

The equations (5.8), (5.9), and (5.11) are the same as the equations (4.14), (4.17), and (4.16) for the $x_{j}$, simply with $x_{j}$ replaced by $d_{j}$. As we have seen in Section 4 , when $n \in E$ and $N=n(n+2)$, the equations for the $x_{j}$ have a solution for all values of $x_{0}$, and hence the equations for the $d_{j}$ have a solution for all values of $d_{0}$. Since a solution $f$ can be multiplied by any non-zero complex number, we may take $d_{0}=1$, and then, considering (4.19), we obtain (5.4).

Thus we see that when $n \geq 0, n \in E$, and $K=(n+1)^{2} / 9$, the equation (1.1) indeed has the solution $f$ given by (5.1) where $c, \zeta$, and $Q$ are as stated, for each $c$ with $c^{3}=-27$. Clearly the three functions $f$ obtained for the three values of $c$ are linearly independent.

The first claim of Theorem 5.1 follows by combining Theorem 1.4 and Theorem 1.1 (taking into account the subsequent remark to this theorem). This completes the proof of Theorem 5.1.

Examples. We consider what the functions $f$ look like for some small values of $n$ when $n \geq 0, n \in E$, and $K=(n+1)^{2} / 9$. Without further indexing, we allow $c$ to be any one of the three values with $c^{3}=-27$. Again, $\zeta=e^{z / 3}$.

We first express solutions $f$ in the style of (1.6). If $n=0$, then $f(z)=\zeta^{-1} e^{c \zeta}$. If $n=1$, then

$$
f(z)=\zeta^{-2}(1-c \zeta) e^{c \zeta}
$$


If $n=4$, then

$$
f(z)=\zeta^{-5}\left(1-c \zeta+\frac{1}{2} c^{2} \zeta^{2}-\frac{1}{7} c^{3} \zeta^{3}+\frac{1}{56} c^{4} \zeta^{4}\right) e^{c \zeta} .
$$

Compare (5.12) to the fact that when $n=4$, we have

$$
Q(z)=1-8 z+28 z^{2}-56 z^{3}+56 z^{4} .
$$

The difference is due to a different coefficient being normalized to be equal to 1 . Apart from the normalizations, the polynomial $Q$ is essentially the reverse (or the reciprocal polynomial) of the polynomial in $c \zeta$ in (5.12).

Regarding the expression (5.1) for the solution $f$, we note that when $n$ gets larger, the coefficients of the polynomial $Q$ become more complicated. For example, if $n=7$, then

$$
Q(z)=1-21 z+210 z^{2}-1330 z^{3}+5880 z^{4}-18200 z^{5}+36400 z^{6}-36400 z^{7} .
$$

If $n=9$, then

$$
\begin{aligned}
Q(z)= & 1-33 z+528 z^{2}-5456 z^{3}+40656 z^{4}-227920 z^{5}+960960 z^{6} \\
& -2932160 z^{7}+5864320 z^{8}-5864320 z^{9} .
\end{aligned}
$$

The formula (5.4) for the coefficients of $Q$ might be used to consider the question mentioned before, see the Remark preceding Theorem 1.4, of whether $\psi$ (or $Q$ ) is related to some known special functions.

\section{References}

[1] BAesch, A.: On the explicit determination of certain solutions of periodic linear differential equations of higher order. - Results Math. 29, 1996, 42-55.

[2] BANK, S.: On the explicit determination of certain solutions of periodic linear differential equations. - Complex Variables Theory Appl. 23, 1993, 101-121.

[3] Bank, S., and I. Laine: On the oscillation theory of $f^{\prime \prime}+A f=0$ where $A$ is entire. - Trans. Amer. Math. Soc. 273, 1982, 351-363.

[4] BAnk, S., and I. LAINE: Representations of solutions of periodic second order linear differential equations. - J. Reine Angew. Math. 344, 1983, 1-21.

[5] Bank, S., I. LAine, and J. LANGLEy: On the frequency of zeros of solutions of second order linear differential equations. - Results Math. 10, 1986, 8-24.

[6] Chinng, Y.M.: On the zero-free solutions of linear periodic differential equations in the complex plane. - Results Math. 38, 2000, 213-225.

[7] Chinng, Y.M., and M. Ismail: On the value distribution theory of second order periodic ODEs, special functions and orthogonal polynomials. - Canad. J. Math. 58, 2006, 726-767.

[8] Chiang, Y. M., I. Laine, and S. WAng: An oscillation result of a third order linear differential equation with entire periodic coefficients. - Complex Variables Theory Appl. 34, 1997, 25-34.

[9] Grosswald, E.: Bessel polynomials. - Lecture Notes in Math. 698, Springer-Verlag, Berlin, 1978.

[10] Hayman, W. K.: Meromorphic functions. - Clarendon Press, Oxford, 1964.

[11] LAIne, I.: Nevanlinna theory and complex differential equations. - Walter de Gruyter, BerlinNew York, 1993.

[12] LANG, S.: Introduction to linear algebra. Second edition. - Springer-Verlag, New York, 1988.

[13] Shimomura, S.: Oscillation results for $n$-th order linear differential equations with meromorphic periodic coefficients. - Nagoya Math. J. 166, 2002, 55-82. 